\title{
Capacidades económicas de la población vulnerable cabeza de hogar y sus miembros mayores de 17 años en el barrio Ciudad Bonita de Soledad Atlántico, 2018
}

\author{
Household heads population's economic capabilities \\ and whose members over 17 years old in Ciudad \\ Bonita of Soledad, Atlántico, 2018 \\ Elkyn Rafael Lugo-Arias iD \\ Corporación Universitaria Minuto de Dios, Barranquilla, Colombia.
}

Luis Fernando Landázury-Villalba iD

Corporación Universitaria Minuto de Dios, Barranquilla, Colombia.

Luis Fernando Escorcia-Márquez iD

Corporación Universitaria Minuto de Dios, Barranquilla, Colombia.

Johanna Orfelina Padilla-Ortiz

Corporación Universitaria Minuto de Dios, Barranquilla, Colombia.

\section{Resumen}

Objetivo: caracterizar la capacidad económica de la población cabeza de hogar y sus miembros mayores de 17 años con situación de vulnerabilidad del barrio Ciudad Bonita de Soledad, Atlántico, para el año 2018. Método: para este estudio se utilizó la teoría de las capacidades y las funciones humanas del nobel de Economía Amartya Sen, aplicada mediante el método de muestreo aleatorio simple, en la cual se realizaron 32 preguntas en las encuestas y entrevistas preestructuradas a 100 jefes de hogar y sus respectivos miembros. Resultados: se encontró que las pocas personas en edad de trabajar, que han decido participar en los programas de capacitación, ofrecidos por las instituciones de formación, de emprendimiento de su propio negocio y del buen manejo de las microfinanzas, el crédito, el ahorro, entre otras variables, han mejorado su situación económica y la calidad de vida en sus hogares, necesarios para la acumulación de conocimiento y la estabilidad en la generación de sus propios recursos o ingresos. Discusiones: Se discute la falta de cobertura y de atención de las instituciones en proyectos productivos con las comunidades por la limitación de su presupuesto. Conclusiones: se propone una mejora de las capacidades económicas que llevan a la prosperidad integral.

Palabras clave: capacidades, funciones, desarrollo económico, desarrollo humano.

Autores de Correspondencia

elkyn.lugo@uniminuto.edu.co llandazuryv@uniminuto.edu.co lescorciama@uniminuto.edu.co jpadillaort@uniminuto.edu.co

Recibido: $12-04-19$

Aceptado: 16-07-19

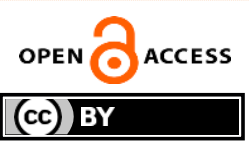

Copyright $(\subset) 2019$

Desarrollo Gerencial

\begin{abstract}
Objective: this paper aims to characterize household heads population's economic capabilities and whose members over 17 years show a social vulnerability status, in Ciudad Bonita neighborhood of Soledad, Atlántico, in 2018. Method: the theory of the capabilities and human functions proposed by Amartya Sen, Nobel Prize in Economics is used in this research; a simple random sampling method, in which 32 questions were asked in prestructured surveys and interviews to 100 household heads involving their members. Results: few people in working age, who have decided to participate in training programs offered by training institutions, entrepreneurship of their own business and the good management of micro finance, credit, savings, among other variables, have improved their economic situation and the quality of life in their homes, necessary for the knowledge accumulation and stability to generate their own resources or income, was found in this research, as result. Discussions: The lack of coverage and attention of the institutions in charge of productive projects, because of their budget limitation. Conclusions: an improvement in economic capacities leading to integral prosperity is proposed.
\end{abstract}

Keywords: capacities, operations, economic development, human development.

JEL Classification: I31, I32, A14, 010.

Como citar este artículo (Apa):

Lugo-Arias, E., Landázury-Villalba, L., Escorcia-Márquez, L. \& Padilla-Ortiz, J. (2019). Capacidades económicas de la población vulnerable cabeza de hogar y sus miembros mayores de 17 años en el barrio Ciudad Bonita de Soledad Atlántico, 2018. Desarrollo Gerencial, 11(2), 1-20. DOI: https://doi.org/10.17081/dege.11.2.3432 


\section{Introducción}

El presente artículo surgió por las necesidades de falta de formación humana y generación de ingresos observadas por la Unidad de Intervención Social de la Universidad Minuto de Dios en las familias vulnerables del barrio Ciudad Bonita de Soledad, Atlántico (Universidad Minuto de Dios, 2016).

La caracterización y el diagnóstico de las capacidades económicas de esta comunidad permitieron la generación de ideas, recomendaciones y propuestas de políticas públicas y privadas con el fin de mejorar las condiciones de vida, mediante la identificación de alternativas productivas, de comercialización, el buen manejo administrativo de los recursos, mediante el ahorro, el emprendimiento y la innovación social, los cuales son un vehículo para reducir los niveles de pobreza multidimensional de estas localidades que se encuentran en vulnerabilidad.

Cuando las localidades acogen esta propuesta de mejorar las alternativas de producción y su administración y distribución, esta decisión puede convertirse en una acción real, que lleva al mejoramiento del bienestar de la comunidad, no solo esperando la ayuda de otras organizaciones, sino tomando la iniciativa de crecer con sus propios recursos, logrando establecer unos indicadores medibles y cuantificables, en cuanto al aumento de los ingresos, de las utilidades, del ahorro y la buena administración de los recursos, que puedan ser objeto de análisis y seguimiento por parte del núcleo de interesados, que les dará una cultura para emprender y administrar su negocio y hogar.

Muchos colombianos de las zonas periféricas y rurales, que viven hoy en día en situación de pobreza extrema en las principales ciudades de Colombia, han sido afectados por los diferentes conflictos sociales presentados durante la historia de la violencia vivida en el país desde la década de 1950 hasta hoy día. Dicha situación ha aumentado el número de las filas de los grupos armados ilegales, los cuales han desplazado forzosamente a las familias de sus tierras, principalmente, a las familias de zonas rurales en la década de 1990, dado que existía muy poca presencia del Estado colombiano y de la justicia que defendiera sus derechos humanos.

Esta situación ha ocasionado que, en la década de 1990, las familias se desplazaran a las zonas marginadas del área metropolitana, cercana a Barranquilla, para buscar mejores condiciones de vida, y así lograr satisfacer sus necesidades básicas y recuperar lo que dejaron o perdieron en sus tierras; dado lo anterior, se vieron obligadas a invadir las comunidades del barrio mencionado en el estudio, estas comunidades son las familias que todavía se encuentran en situación de pobreza, en el barrio Ciudad Bonita del municipio de Soledad, Atlántico, estableciéndose en este con el fin de empezar una nueva vida con sus familias.

Los habitantes de este barrio se encuentran dentro de los límites esperados de pobreza, casi absoluta, sin lograr tener objetivos claros, en cuanto a sus metas a corto, mediano y a largo plazo, por lo cual realizar esta propuesta permitirá conocer su diagnóstico socioeconómico, para proponer alternativas de mejoramiento de los ingresos y de la calidad de vida de sus habitantes, mediante la identificación y establecimiento de estrategias y planes de acción adecuados, los cuales generen una cultura en cuanto a la creación de alternativas productivas, que capacite a los habitantes de los barrios intervenidos y que sepan articularse en el mercado y comercializar sus productos, utilizando la gestión del conocimiento, la ciencia, la tecnología, la innovación social, para vender por medio de la Internet y los aplicativos diseñados por el Ministerio de Agricultura del Gobierno, tales como Agronet, entre otros, de tal manera que aumenten sus clientes, pedidos, cargas, sus ingresos y puedan mejorar su calidad de vida y las de sus familias. 
Dada la situación anterior, mediante este estudio se requiere identificar las ventajas y las fortalezas de las diferentes actividades productivas, puntualizando procesos que les orienten a dinamizar un establecimiento socioeconómico, llevando esta propuesta como proyecto bajo esquemas productivos que ayuden a visualizar las alternativas productivas, las cuales puedan garantizar su seguridad alimentaria, su autosostenibilidad y su desarrollo psicosocial y familiar. Ante este contexto surgieron las siguientes preguntas de investigación: ¿Cuál es la situación y el nivel de las capacidades productivas que presenta la población vulnerable cabeza de hogar y sus miembros mayores de 17 años en el barrio Ciudad Bonita, 2018? ¿Qué sugerencias o recomendaciones se pueden establecer para mejorar su situación socioeconómica, desde el punto de vista productivo, emprendedor y de innovación social?

Por tanto, esta investigación tuvo como objetivo principal caracterizar la capacidad económica de las comunidades en situación de pobreza del barrio Ciudad Bonita de Soledad Atlántico.

El presente trabajo está organizado de la siguiente manera: en la primera sección, se muestra la fundamentación teórica de las capacidades económicas y funciones humanas, seguido del diseño metodológico, la caracterización de la situación social en educación, salud y capacidades productivas, económicas y de ciencia, tecnología e innovación en las personas en edad de trabajar del barrio Ciudad Bonita. Por último, se derivan las conclusiones, las discusiones y las recomendaciones para mejorar las alternativas y decisiones productivas de políticas públicas y de las capacidades económicas de estas familias.

\section{Fundamentación teórica}

\section{El enfoque de capacidad de Sen.}

El enfoque de la capacidad es un marco evaluativo para el bienestar individual. Los conceptos centrales son funciones y capacidades. Sen (1995):

Define un funcionamiento como un logro de una persona, es decir, lo que logra hacer o ser. Los funcionamientos comprenden las actividades y estados de ser de un individuo, por ejemplo, gozar de buena salud, estar bien protegido, moverse libremente o ser educado. La capacidad es una noción derivada y refleja los diversos funcionamientos que él o ella puede lograr, e implica la libertad de la persona para elegir entre diferentes formas de vida. (p. 125)

El enfoque de la capacidad para el bienestar humano es una "concentración en la libertad de alcanzar en general y las capacidades para funcionar en particular", y los conceptos centrales de este enfoque son "funciones y capacidades". (Sen, 1995, p. 266). "Un funcionamiento es un logro, mientras que una capacidad es la capacidad de lograr" (Sen, 1987, p. 36). Antes de analizar en qué consiste más detalladamente el enfoque de capacidad, sería útil comprender cómo llegó Sen a desarrollar este enfoque. A partir de la década de 1970, Sen y sus asociados comenzaron a elaborar una crítica de la economía y el utilitarismo del bienestar general, y extendieron y modificaron un marco tradicionalmente utilizado en microeconomía, que describe cómo los individuos obtienen ingresos y bienestar. 
Según Sen (1990):

Las capacidades comprenden lo que una persona es capaz de hacer o ser: la capacidad de estar bien alimentada, de evitar la morbilidad o la mortalidad evitable, de leer, escribir y comunicarse, de participar en la vida de la comunidad, de aparecer en público sin vergüenza, más allá de los enfoques tradicionales de bienestar. (p. 126)

Para comprender el enfoque de capacidad de Sen, que proporciona el marco más completo para conceptualizar el bienestar, es esencial examinar cómo analiza lo incompleto de los conceptos tradicionales de bienestar: el enfoque de la mercancía o el ingreso y la utilidad.

A nivel internacional existen varios estudios que explican la situación socioeconómica de la baja capacidad humana en la generación de ingresos y la problemática de pobreza en diferentes países, y sus regiones. Como es el caso de Sen (1995), quien explicó que la pobreza no solo se caracteriza por tener más o menos ingresos, pues este es solo un aspecto de las carencias, que debe complementarse con el análisis de las potencialidades individuales, mediante su teoría de las capacidades (capabilities) y de conjuntos de atributos personales socialmente viables y deseables, funciones institucionales (functionings), desde un enfoque de capacidades y derechos, en donde logró evaluar y validar el bienestar y la libertad de las personas en Bangladesh, India y otras comunidades (Sugden, 1993; Pettit, 2001; Robeyns, 2005; Stiglitz, Sen \& Fitoussi, 2010; Nussbaum, 2011; Wells, 2012; Bravo, Schwartz, \& Drèze 2014; Michalos, 2017; Arrow, Bowles \& Durlauf, 2018).

Los indicadores de capacidades productivas y económicas de las poblaciones vulnerables se observan con las condiciones sociales de acceso al conocimiento, a la salud, el empleo, emprendimiento, distribución de los recursos, a la participación igualitaria de género o de la población en las instituciones, que fomentan e impulsan el desarrollo humano, según la teoría de Sen (1995), como estrategias para enfrentar el flagelo de la pobreza como un problema o fenómeno económico y social.

Según Sen (2018), el enfoque en la utilidad o los recursos puede ser engañoso, ya que lo esencial no es la suma total de los productos, sino lo que estos hacen a las personas. Al centrarse en la libertad, Sen (1985) sostiene que el enfoque de capacidad reconoce que las personas difieren en su capacidad para convertir bienes en logros valiosos, debido a factores personales y de ubicación, y a los acuerdos sociales.

\section{El enfoque de la capacidad como marco evaluativo.}

Varios académicos han adoptado las ideas de Sen y las han desarrollado en el enfoque de capacidades, como un intento de desarrollar un marco normativo amplio para la evaluación del bienestar individual y los arreglos sociales (Nussbaum, 2011; Robeyns, 2005 \& Sen, 2018). La característica principal del enfoque de capacidad es alejarse de los métodos de evaluación basados en los ingresos y centrarse en la capacidad de las personas para lograr lo que ellos valoran. Por lo tanto, el bienestar puede medirse evaluando la libertad y las opciones de las personas, en lugar de su ingreso o consumo.

La capacidad es el espacio informativo para hacer juicios evaluativos. Según Sen (2018), el enfoque de capacidades amplía el espacio informativo para hacer juicios evaluativos, al reconocer la naturaleza multidimensional 
Elkyn Lugo-Arias, Luis Fernando Landázury-Villalba, Luis Escorcia-Márquez, Johanna Padilla-Ortiz

del bienestar humano. En el campo del desarrollo, muchos otros enfoques se han alejado de la definición de pobreza basada en los ingresos, al incluir las percepciones de las personas y aceptar las múltiples facetas de la pobreza. Sin embargo, argumentan que estos no captan la justificación filosófica del enfoque de capacidad, en cuyo núcleo se basa el concepto de que el desarrollo consiste en proporcionar condiciones que faciliten la capacidad de las personas para llevar una vida floreciente.

Ponthieux y Concialdi (2001), los cuales realizaron un estudio denominado Bajos salarios y trabajadores pobres: una comparación entre Francia y Estados Unidos, donde utilizaron el salario promedio de la población, según su actividad para medir y comparar la pobreza entre Estados Unidos y Francia, durante el periodo 1983-1999, encontrando que la parte de asalariados con bajos salarios de Estados Unidos es dos veces superior que en Francia, los cuales se caracterizan por ser más pobres laboralmente. Las mujeres, los jóvenes y los menos cualificados con menores ingresos o pobres se ven más que proporcionalmente afectados, dado que solo trabajan tiempo parcial. En Estados Unidos los pobres en edad de trabajar tienen mayor probabilidad de ser empleados que en el caso francés. En ambos países hay un vínculo claro entre el riesgo de pobreza y el número anual de horas prestadas.

Otro estudio reciente es el de Henninger y Snel (2002), quienes en su estudio relacionado con el impacto de los mapas de pobreza evaluaron un número importante de estudios de caso, dentro de los cuales se destacan varios países latinoamericanos, africanos y asiáticos.

Autores como Quintero y Mejía (2006), en su estudio, desarrollaron pobreza subjetiva, como línea de análisis alternativa, que les permitió evaluar la percepción que tienen los seres humanos frente a la pobreza, a través de un análisis complementario, que permitió caracterizar el fenómeno y la construcción de políticas eficientes y adecuadas para combatir la pobreza, mediante la realización de encuestas de percepción, analizándose desde un punto subjetivo, dejando como resultado la reducción de las adversidades económicas, sociales y culturales, que provoca la situación de pobreza entre las personas que la padecen en el presente, para que no les transfieran estas adversidades a las generaciones futuras.

En Colombia, se destacan los siguientes estudios sobre los factores económicos determinantes de la pobreza:

Núñez y Ramírez (2002) quienes analizan los determinantes macroeconómicos de la pobreza en el país, incluyendo variables macroeconómicas relacionadas con las dotaciones y la situación laboral de los individuos. En este último enfoque, Browning y Lusardi (1996) y Lustig y Székely (1998) sostienen que la intensidad de la pobreza está fuertemente asociada a la falta de acceso a los activos físicos, financieros y de capital humano.

Botello y Quintero (2012), por su parte, realizaron un estudio de las condiciones de vida de las personas a través del Índice de Calidad de Vida y la relación con los indicadores subjetivos de bienestar (la felicidad, la satisfacción y la autopercepción de pobreza) en el que hallaron que existen diferencias en las condiciones de bienestar entre las regiones del país. Por ejemplo, encontraron que las zonas como Bogotá, Valle del Cauca y San Andrés Isla, presentan mejores indicadores de bienestar y felicidad, mientras que en la región Caribe y Pacifica, estos indicadores son los más bajos. Esto demuestra que el indicador de la felicidad no es medida por factores materiales como en otras comunidades y que la toma de decisiones está relacionada con la dignidad, el barrio y la capacidad de ayudar a los demás. 


\section{Método}

\section{Diseño.}

Con base en el marco teórico que soporta esta investigación y los objetivos que se plantearon, se procedió al desarrollo de estos últimos, mediante un estudio descriptivo de las características que identifican nuestro contexto investigativo, con base en un enfoque mixto, mediante datos cuantitativos y cualitativos de fuente primaria con la participación activa de las familias focalizadas en el barrio Ciudad Bonita. Este barrio se encuentra ubicado en el área metropolitana de la ciudad de Barranquilla, cuenta con población de 100 familias y su estratificación es uno (1) bajo.

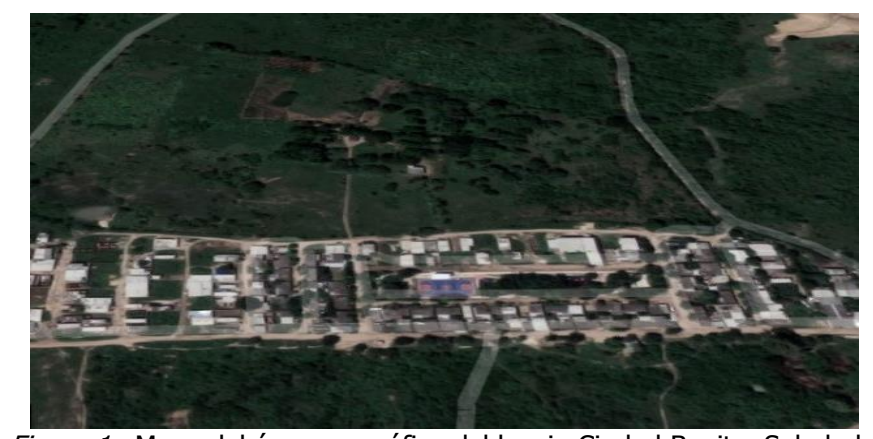

Figura 1. Mapa del área geográfica del barrio Ciudad Bonita, Soledad.

Nota. Fuente: Google mapas (2018)

Se emplearon criterios teóricos y técnicos, indicadores de capacidades económicas y humanas con las dimensiones de las condiciones sociales de acceso al conocimiento, a la educación, a la formación, a la salud, a la tecnología, la generación de ingresos, la participación igualitaria de género en la población, según la teoría de Amartya Sen, relacionados al de estudio de la pobreza como un problema o fenómeno económico y social, adecuados para el análisis de los datos, mediante instrumentos, herramientas y argumentos necesarios para caracterizar las capacidades y funciones humanas en las familias en situación de pobreza de las poblaciones asentadas en el barrio de estudio.

\section{Participantes.}

A lo largo del desarrollo de este trabajo, se fueron descubriendo particularidades del contexto territorial en la población en edad de trabajar del barrio Ciudad Bonita, ya que las actuales condiciones de capacidades y funciones humanas y productivas ameritan la participación activa de las personas en edad de trabajar, jefes de hogar y las instituciones del Estado, en establecer estrategias de política pública para potencializar y fortalecer las capacidades de la comunidad, que le permitan generar sus propios ingresos sosteniblemente y acumular recursos productivos.

Además, se utilizaron como fuentes primarias para la recolección de la información, la encuesta, en una población de 130 jefes o cabezas de hogar, uno por cada hogar del barrio Ciudad Bonita, los cuales sostienen económicamente el hogar. Para calcular la muestra, se utilizó la siguiente fórmula de aleatoriedad simple:

$$
n=\frac{Z^{2} * P * Q * N}{N * E^{2}+Z^{2} * P * Q}
$$


Donde:

$\mathrm{n}$ : tamaño de la muestra

Z: nivel de confianza $(1,96)$

p: variabilidad positiva $(0,5)$

q: variabilidad negativa $(0,5)$

$\mathrm{N}$ : tamaño de la población objetivo del barrio Ciudad Bonita (130 hogares)

E: porcentaje de error $(0,05)$

Muestra de hogares de Ciudad Bonita:

$$
\begin{gathered}
n=\frac{1.96^{2} * 0.5 * 0.5 * 130}{130 * 0.05^{2}+1.96^{2} * 0.5 * 0.5} \\
n=100
\end{gathered}
$$

Para ello, se aplicaron en total 100 encuestas a los jefes de cada hogar y a sus miembros mayores de 17 años, como muestra de la población para caracterizar las capacidades económicas del barrio Ciudad Bonita, a las cuales se les realizó su formulación y tabulación.

\section{Instrumentos de recolección de información y procedimiento.}

Se empleó la encuesta y la entrevista estructurada, con el propósito de conocer la percepción que tenían las personas frente al tema.

El método de la observación estructurada es un mecanismo de visualización directa, la cual consistió en utilizar la vista como una guía referencial para palpar y constatar el fenómeno en estudio de forma precisa.

Este proceso tuvo como objetivo corroborar algunos de los aspectos sociales, económicos y productivos, obtenidos a partir de las entrevistas y cuestionarios, realizadas con evidencias, usando papel, lápiz, cámara fotográfica, grabadora digital de voz y cámara de video.

\section{Resultados}

\section{Capacidades productivas y económicas.}

La caracterización, propuesta y recomendación de las capacidades productivas, en esta población, busca es estructurar las diversas posibilidades de fortalecimiento al mejoramiento de la calidad de vida de la población, mediante la formulación de estrategias y asesorías, que le sirvan a estas familias para mejorar e innovar en sus labores productivas, su cultura de ahorro y emprendimiento, permitiendo tener negocios con ingresos sostenibles y que generen empleo e ingresos, con metas cuantificables y medibles (Landázury, Lechuga, Ferrer \& Prieto, 2017; Lugo, Sáenz \& Lugo, 2018; Lugo \& Lugo, 2018; Lugo, et al., 2018; Landázury et al., 2018 \& Henríquez et al., 2018). Sin embargo, en el análisis que se hizo a esta comunidad del barrio Ciudad Bonita, se pudo detectar que los pocos negocios no tienen el crecimiento deseado. 
Para mejorar el bienestar y la calidad de vida de estas poblaciones vulnerables, se debe proponer un aumento en el conocimiento de las buenas prácticas de las operaciones productivas, para su mayor aprovechamiento, así mismo, deben mejorar sus condiciones económicas que impiden acceder a entes capacitadores.

Al implementar las alternativas productivas en esta comunidad, la economía comienza a beneficiar a toda la comunidad en general, a cambiar la calidad de vida personal, familiar, social y ambiental, preparándolos a través del conocimiento a los diversos cambios que la sociedad los conduce continuamente, llevando a las localidades a un nivel sociocultural y socioeconómico estable y en avance (Lugo et al., 2018).

\section{Caracterización de la situación social en educación, salud y capacidades productivas.}

Tabla 1.

Sexo de los miembros del grupo familiar

$\begin{array}{ccc}\text { Sexo } & \text { Personas mayores de } 17 \text { años } & \text { Part. (\%) } \\ \text { Masculino } & 104 & 47.06 \% \\ \text { Femenino } & 117 & 52.94 \% \\ \text { Total } & 221 & 100.00 \%\end{array}$

Fuente: elaboración propia mediante encuesta (2018).

En la tabla 1, se puede apreciar que de las 221 personas mayores de 17 años de edad, de los 100 hogares: el $52.94 \%$ es de género femenino y el $47.06 \%$ es masculino.

Tabla 2.

Estado civil de las personas mayores de 17 años del grupo familiar

\begin{tabular}{ccc}
\hline Estado Civil & Personas mayores de 17 años & Part. (\%) \\
\hline Separado & 6 & $2.71 \%$ \\
\hline Soltero & 114 & $51.58 \%$ \\
Casado & 40 & $18.10 \%$ \\
\hline Unión libre & 58 & $26.24 \%$ \\
\hline Viudo & 3 & $1.36 \%$ \\
\hline Total & 221 & $100 \%$ \\
\hline
\end{tabular}

En la tabla 2, se observa que de las 221 personas mayores de 17 años de edad: 114 personas, que corresponden al $51.58 \%$, se encuentran solteros, lo cual es un porcentaje considerablemente alto y esto se puede explicar a la falta de ingresos para sostener un matrimonio y una familia, también debido a que hay familias donde los padres niegan su estado civil de compromiso y la separación provoca problemas intrafamiliares o psicológicos. Así mismo, el 26.24\% viven en unión libre y el $18.10 \%$ están casados.

Tabla 3.

Conformación de las personas mayores de 17 años del grupo familiar Conformación del grupo familiar Jefe de familia Personas mayores de 17 años

Cónyuge $\quad 35$

Hermano(a) 15

Padre/madre $\quad 24$

Hijo(a) $\quad 80$

Total 221

Fuente: elaboración propia mediante encuesta (2018). 
En la tabla 3, se observa que de las 221 personas mayores de 17 años: 80 son hijos, 67 son jefes de familia, 35 son cónyuges, 15 son hermanos y 24 son entre padres y madres de familia.

Tabla 4.

Ocupación de las personas mayores de 17 años del grupo familiar

$\begin{array}{ccc}\text { Ocupación } & \text { Personas mayores de } 17 \text { años } & \text { Part. (\%) } \\ \text { Independiente } & 31 & 14.03 \% \\ \text { Jubilado } & 8 & 3.62 \% \\ \text { Ama de casa } & 57 & 25.79 \% \\ \text { Empleado } & 39 & 17.65 \% \\ \text { Desempleado } & 30 & 13.57 \% \\ \text { Estudiante } & 56 & 25.34 \% \\ \text { Total } & 221 & 100 \%\end{array}$

Fuente: elaboración propia mediante encuesta (2018).

En la tabla 4, se observa que de las 221 personas mayores de 17 años: solo el $14 \%$ trabajan de manera independiente y solo el $17.65 \%$ labora como empleado en una empresa. Por su parte, los jubilados son solamente el $3.62 \%$ de esta población, el resto de las personas, correspondiente al $74 \%$, en los cuales se desempeñan como estudiante, ama de casas y algunos están desempleados, esta última ocupación es alta, con un $13.57 \%$, porcentaje que ubica este indicador por encima del promedio departamental y nacional. La participación más alta fue la de ama de casa y estudiante, cada una por encima del $25 \%$, lo cual refleja que los estudiantes jóvenes y solteros son los que más se capacitan, se dedican más al estudio, mientras que la mujer ama de casa poco se capacita y se dedica al cuidado de sus hijos, esposo u otro familiar, y esto debido a que cuenta con la ayuda del jefe de hogar.

Tabla 5.

Nivel de escolaridad de las personas mayores de 17 años de edad

\begin{tabular}{|ccc|}
\hline Nivel de escolaridad & Personas mayores de 17 años & Part. (\%) \\
\hline Secundaria completa & 57 & $25.79 \%$ \\
\hline Secundaria incompleta & 36 & $16.29 \%$ \\
\hline Primaria completa & 54 & $24.43 \%$ \\
\hline Universitaria & 21 & $9.50 \%$ \\
\hline Técnica o tecnológica & 13 & $5.88 \%$ \\
\hline Lee y escribe & 22 & $9.95 \%$ \\
\hline Analfabeta & 18 & $8.14 \%$ \\
\hline Total encuestados & 221 & $100 \%$ \\
\hline
\end{tabular}

Fuente: elaboración propia mediante encuesta (2018).

En la tabla 5, se puede apreciar que de las 221 personas mayores de 17 años: 40 de ellas, que son más del $18 \%$, no han estudiado ni siquiera la primaria, de los cuales el $8.14 \%$ es analfabeta y el 9.95\% por lo menos aprendió a leer y escribir por su cuenta, mientras que 90 personas, es decir el $41 \%$ terminó la primaria, el $25.79 \%$ la secundaria, el $5.88 \%$ carreras técnicas y tecnológicas y el $9.5 \%$ son universitarios.

Tabla 6.

Tipo de afiliación en salud de las personas mayores de 17 años

\begin{tabular}{ccc} 
Tipo de afiliación en salud & Personas mayores de 17 años & Part. (\%) \\
\hline Contributivo & 41 & $18.55 \%$ \\
Subsidiado & 180 & $81.45 \%$ \\
Total encuestados & 221 & $100 \%$
\end{tabular}

Fuente: elaboración propia mediante encuesta (2018). 
En la tabla 6, se tiene que el $81.45 \%$ de las 221 personas mayores de 17 años están afiliadas en el régimen de salud subsidiado con estrato 1 , el resto se encuentra vinculado al régimen contributivo (18.55\%).

Tabla 7.

Discapacidad de las personas mayores de 17 años

\begin{tabular}{|ccc|}
\hline Discapacidad & Personas mayores de 17 años & Part. (\%) \\
\hline Visual & 3 & $1.36 \%$ \\
\hline Motora & 21 & $9.50 \%$ \\
\hline Del habla & 3 & $1.36 \%$ \\
\hline Mental & 13 & $5.88 \%$ \\
\hline Auditiva & 3 & $1.36 \%$ \\
\hline Ninguna & 178 & $80.54 \%$ \\
\hline Total encuestados & 221 & $100 \%$ \\
\hline
\end{tabular}

Fuente: elaboración propia mediante encuesta (2018).

En la tabla 7, la población mayor de 17 años del barrio Ciudad Bonita, en su mayoría el 80.54\%, no tiene ningún problema de discapacidad que le impida realizar una actividad productiva, sin embargo, el $9.50 \%$ tiene limitaciones motoras, el $5.88 \%$ limitaciones mentales, mientras que las demás limitaciones, como la visión, la audición y el habla, fueron de $1.36 \%$ cada una, es decir, 3 personas en cada limitación.

Tabla 8.

Nivel educativo del jefe de hogar

\begin{tabular}{ccc}
\hline Nivel educativo del jefe de hogar & Jefe de hogar & Part. (\%) \\
\hline Primaria & 78 & $78 \%$ \\
\hline Bachiller & 9 & $9 \%$ \\
\hline Técnico & 1 & $1 \%$ \\
\hline Pregrado & 0 & $0 \%$ \\
\hline Ninguno & 12 & $12 \%$ \\
\hline Total encuestados & 100 & $100 \%$ \\
\hline Fuente: elaboración propia mediante encuesta (2018).
\end{tabular}

En la tabla 8, se observa que ningún jefe de hogar tiene un nivel de educación alto o profesional, solo un $1 \%$ es técnico, un $9 \%$ es bachiller y un $78 \%$ estudió hasta la primaria, sin embargo, la tasa de analfabetismo es muy alta, la cual es del $12 \%$ del total de encuestados.

Tabla 9.

Percepción de la necesidad de capacitarse ¿Considera que necesita capacitarse? Jefe de hogar Part. (\%)

$\begin{array}{ccc}\text { Sí } & 79 & 79 \% \\ \text { No } & 21 & 21 \% \\ \text { encuestados } & 100 & 100 \%\end{array}$

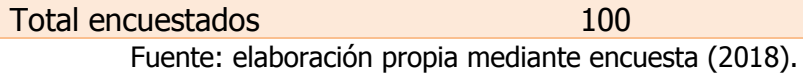

Según la tabla 9, el $79 \%$ de los jefes de hogar consideran que necesitan capacitarse y están dispuestos a prepararse, mientras que el $21 \%$ no tiene el deseo de hacerlo. 
Tabla 10.

Beneficios de los programas de capacitación del Estado ¿Ha sido beneficiario de los Jefe de hogar Part. (\%) programas de capacitación del estado?

\begin{tabular}{ccc|}
\hline Sí & 11 & $11 \%$ \\
\hline No & 89 & $89 \%$ \\
\hline Total encuestados & 100 & $100 \%$ \\
\hline
\end{tabular}

Fuente: elaboración propia mediante encuesta (2018).

En la tabla 10, el $89 \%$ de los jefes de hogar no reciben ningún beneficio de los programas de capacitación del Estado, mientras que el $11 \%$ sí ha recibido.

Tabla 11.

Percepción en la elección de las áreas de capacitación Áreas en la que le gustaría capacitarse Ventas (comercial) Salud Jefe de hogar Part. (\%)

\begin{tabular}{|ccc|}
\hline Areas en la que le gustaria capacitarse & Jefe de hogar & Part. (\%) \\
\hline Ventas (comercial) & 24 & $24 \%$ \\
\hline Salud & 0 & $0 \%$ \\
\hline Agropecuario & 4 & $4 \%$ \\
\hline Administración de negocios & 35 & $35 \%$ \\
\hline Industrial & 3 & $3 \%$ \\
\hline Educación & 11 & $11 \%$ \\
\hline Belleza & 23 & $23 \%$ \\
\hline Total encuestados & 100 & $100 \%$ \\
\hline Fuente: elaboración propia mediante encuesta (2018).
\end{tabular}

Según la tabla 11, a la mayoría de los 100 jefes de hogar encuestados, les gusta capacitarse y están interesados en estudiar: el $35 \%$ en administración de negocios, el $24 \%$ en ventas, el $23 \%$ en belleza, el $11 \%$ en educación, el $4 \%$ en lo agropecuario y el $3 \%$ en lo industrial. En cuanto a la educación, la mayoría de los participantes hizo referencia a terminar sus estudios de administración de negocios para poder obtener su negocio en casa.

Tabla 12.

Percepción de las condiciones de vida del jefe de hogar ¿Cómo cree que serán las condiciones de Jefe de hogar Part. (\%) vida de usted y su familia en un año?

\begin{tabular}{ccc|}
\hline Seguirán siendo iguales & 12 & $12 \%$ \\
\hline Mejoraran & 80 & $80 \%$ \\
\hline Empeoraran & 8 & $8 \%$ \\
\hline Total encuestados & 100 & $100 \%$ \\
\hline
\end{tabular}

Fuente: elaboración propia mediante encuesta (2018).

Según la tabla 12, la mayoría de los jefes de hogar, es decir el $80 \%$, se encuentran optimistas, creen que su calidad de vida y el bienestar de su familia mejorará para el próximo año, el $12 \%$ piensa que seguirá igual y solo el $8 \%$ cree que empeorará. Estos últimos necesitan motivación y mayores capacidades productivas para mejorar sus condiciones de vida. 


\section{Caracterización de la situación económica y financiera de la familia.}

Tabla 13.

Tipo de generación de ingresos del jefe de hogar

\begin{tabular}{ccc}
\hline $\begin{array}{c}\text { ¿De qué manera se generan } \\
\text { los ingresos de esta familia }\end{array}$ & Jefe de hogar & Part. (\%) \\
\hline Trabajo formal & 34 & $34 \%$ \\
\hline Independiente & 39 & $39 \%$ \\
\hline Aporte familiar & 3 & $3 \%$ \\
\hline Subsidio & 13 & $13 \%$ \\
\hline Pensión & 11 & $11 \%$ \\
\hline Total encuestados & 100 & $100 \%$ \\
\hline Fuente: elaboración propia mediante encuesta (2018).
\end{tabular}

La tabla 13, evidencia que la mayoría de los jefes de hogar (39\%) genera los ingresos con su trabajo independiente, el 34\% con su trabajo formal en empresas, el 13\% por subsidios, el $11 \%$ por pensión y el $3 \%$ por ayuda de familiares. Esto muestra que todos requieren mejorar sus ingresos por medio del fortalecimiento de las capacidades productivas y el emprendimiento.

Tabla 14.

Subsidios de los miembros del grupo familiar

\begin{tabular}{ccc}
\hline $\begin{array}{c}\text { ¿Ha recibido o recibe alguno de los } \\
\text { siguientes subsidios? }\end{array}$ & Miembros & Part. (\%) \\
\hline Jóvenes en acción & 8 & $18.18 \%$ \\
\hline Adulto mayor & 4 & $9.09 \%$ \\
\hline Familias en acción & 24 & $54.55 \%$ \\
\hline Desplazados & 7 & $15.91 \%$ \\
\hline Red unidos & 1 & $2.27 \%$ \\
\hline Organizaciones No Gubernamentales & 0 & $0 \%$ \\
\hline Iglesia & 0 & $0 \%$ \\
\hline Total encuestados & 44 & $100 \%$ \\
\hline Fuente: elaboración propia mediante encuesta (2018).
\end{tabular}

En la tabla 14, el jefe de hogar respondió que en su núcleo familiar solamente 44 personas reciben subsidios, siendo principalmente: $54.55 \%$ de Familias en Acción (padres de niños), 18.18\% Jóvenes en Acción (estudiantes de secundaria), $15.91 \%$ desplazados (víctimas), 9.09\% adulto mayor y $2.27 \%$ Red Unidos (población en pobreza extrema).

Tabla 15.

Trabajo lucrativo o negocio económico en la vivienda

\begin{tabular}{ccr}
\hline $\begin{array}{c}\text { ¿En su vivienda se realiza algún trabajo o } \\
\text { negocio que genere ingresos económicos? }\end{array}$ & Jefe de hogar & Part. (\%) \\
\hline Sí & 28 & $28 \%$ \\
\hline No & 72 & $72 \%$ \\
\hline Total encuestados & 100 & $100 \%$ \\
\hline
\end{tabular}

Fuente: elaboración propia mediante encuesta (2018).

La tabla 15, muestra que el $72 \%$ de los jefes de hogar no realiza ningún negocio que le genere un ingreso adicional, mientras que solo el $28 \%$ sí cuentan negocios que les generan ingresos adicionales. Esto refleja que los jefes de hogar y sus familias necesitan motivación y mayores capacidades productivas para mejorar sus condiciones de vida. 
Tabla 16.

Ingresos promedios mensuales de los jefes de hogar

\begin{tabular}{|c|c|c|}
\hline $\begin{array}{l}\text { ¿Cuáles son los ingresos } \\
\text { mensuales promedio de la familia? }\end{array}$ & Jefe de hogar & Part. (\%) \\
\hline Menos de $1 *_{\text {SMLV }}$ & 13 & $13 \%$ \\
\hline $1 *_{S M L V}$ & 76 & $76 \%$ \\
\hline Más de $1 *_{\text {SMLV }}$ & 11 & $11 \%$ \\
\hline Total encuestados & 100 & $100 \%$ \\
\hline
\end{tabular}

En la tabla 16 , se puede observar que el $76 \%$ de los jefes de hogar tienen ingresos equivalentes a un salario mínimo, el $13 \%$ gana menos de un salario mínimo y tan solo el $11 \%$ gana un poco más del salario mínimo. Esto muestra que todos necesitan generar más ingresos, por medio del fortalecimiento de las capacidades productivas y el emprendimiento.

Tabla 17.

Reporte de la vida crediticia del jefe de hogar en centrales de riesgo ¿Se encuentra reportado en alguna central de riesgo? Jefe de hogar Part. (\%)

\begin{tabular}{|ccc|}
\hline Sifín & 5 & $5 \%$ \\
\hline Data crédito & 28 & $28 \%$ \\
\hline Covinoc & 0 & $0 \%$ \\
\hline Otra & 0 & $0 \%$ \\
\hline Ninguna & 67 & $67 \%$ \\
\hline Total encuestados & 100 & $100 \%$ \\
\hline
\end{tabular}

Fuente: elaboración propia mediante encuesta (2018).

La tabla 17 muestra que es alta la cantidad de jefes de hogares que están endeudados, siendo los reportados en la central de riesgo data crédito, el $28 \%$ y los reportados en la Sifín el $5 \%$, para un total de $33 \%$ de personas con mala hoja de vida crediticia. Por su parte, el $67 \%$ restante, que representa la mayoría de los jefes de hogar, no tienen ninguna deuda. Esto muestra que todos necesitan generar más ingresos, por medio del fortalecimiento de las capacidades productivas y el emprendimiento.

Tabla 18.

Vinculación con entidades financieras de microcrédito

\begin{tabular}{ccc}
\hline $\begin{array}{c}\text { ¿Tiene vínculo con alguna entidad financiera } \\
\text { de microcrédito? }\end{array}$ & Jefe de hogar & $\begin{array}{c}\text { Part. } \\
(\%)\end{array}$ \\
\hline Bancamía & 5 & $5 \%$ \\
\hline Banco de Oportunidades & 0 & $0 \%$ \\
\hline Banco privado & 0 & $0 \%$ \\
\hline Paga diario & 3 & $3 \%$ \\
\hline Otro & 0 & $0 \%$ \\
\hline Ninguna & 92 & $92 \%$ \\
\hline Total encuestados & 100 & $100 \%$ \\
\hline Fuente: elaboración propia mediante encuesta (2018).
\end{tabular}

En la tabla 18, se muestra que es baja la cantidad de jefes de hogares que tienen microcréditos, como apalancamiento para sus negocios, siendo su vinculación con las siguientes entidades: Bancamía (5\%) y paga diario (3\%), para un total de $8 \%$ de las personas. Por su parte, el $92 \%$ restante no tienen ninguna vinculación con alguna 
entidad financiera. Esto muestra que estas personas requieren comprometerse y arriesgarse a financiar, para capacitarse, emprender y generar más ingresos y sus negocios propios.

Tabla 19.

Vinculaciones con Fundaciones o Cooperativas

\begin{tabular}{ccc}
\hline $\begin{array}{c}\text { ¿Se encuentra vinculado con alguna } \\
\text { fundación o cooperativa? }\end{array}$ & Jefe de hogar & Part. (\%) \\
\hline Fundación Mario Santodomingo & 8 & $8 \%$ \\
\hline Fundación Mundo Mujer & 3 & $3 \%$ \\
\hline Famiempresa & 0 & $0 \%$ \\
\hline Actuar & 0 & $0 \%$ \\
\hline Otra & 0 & $0 \%$ \\
\hline Ninguna & 89 & $89 \%$ \\
\hline Total encuestados & 100 & $100 \%$ \\
\hline
\end{tabular}

Fuente: elaboración propia mediante encuesta (2018).

En la tabla 19 se puede observar que es baja la cantidad de jefes de hogares que se encuentran vinculadas a fundaciones o cooperativas de desarrollo y de capacidad humana, siendo su vinculación con las siguientes entidades: Fundación Mario Santodomingo (8\%) y Fundación Mundo Mujer (3\%), para un total de 11\% de las personas. Por su parte, el $89 \%$ restante no tienen ninguna vinculación con alguna fundación o cooperativa. Esto muestra que estas personas necesitan motivarse, relacionarse y participar en estas organizaciones para generar mayores capacidades productivas, tener mayor apoyo social y económico, que le permitan mejorar sus condiciones de vida.

Tabla 20.

Estrategia de ahorro del jefe de hogar

\begin{tabular}{ccc}
\hline ¿Posee alguna estrategia de ahorro? & Jefe de hogar & Part. (\%) \\
\hline Personal & 26 & $26 \%$ \\
\hline Entidad & 2 & $2 \%$ \\
\hline Grupo de ahorro & 6 & $6 \%$ \\
\hline Ninguna & 66 & $66 \%$ \\
\hline Total encuestados & 100 & $100 \%$ \\
\hline
\end{tabular}

Fuente: elaboración propia mediante encuesta (2018).

En la tabla 20 de puede apreciar poco la cantidad de jefes de hogares que ahorran, siendo sus ahorros solamente el $34 \%$, de los cuales el $26 \%$ lo hace usando estrategias personales y guardando su dinero en su casa, mientras que solamente el $2 \%$ lo hace en una entidad financiera y el $6 \%$ en un grupo de ahorro. Por su parte, el $66 \%$ restante no ahorra ni utiliza estrategia de ahorro. Esto demuestra que estas personas necesitan capacitarse más en administración y finanzas para que puedan aprender a tomar decisiones con base en estrategias ganadoras, invertir mejor, tener recursos, activos y capital de trabajo para emprender en negocios, que le generen mayor margen de utilidad y rentabilidad.

Tabla 21.

Percepción acerca del emprendimiento de un negocio

\begin{tabular}{ccc}
\hline ¿Le gustaría emprender un negocio? & Jefe de hogar & Part. (\%) \\
\hline Sí & 78 & $78 \%$ \\
\hline No & 22 & $22 \%$ \\
\hline Total encuestados & 100 & $100 \%$ \\
\hline
\end{tabular}

Fuente: elaboración propia mediante encuesta (2018). 
La tabla 21 muestra que a la mayoría de los jefes de hogares (78\%) les gustaría emprender sus propios negocios, esto requiere la identificación de las actividades productivas en las cuales el gobierno y las organizaciones o instituciones sociales y económicas de apoyo deben intervenir para generar un impacto en el desarrollo socioeconómico de esta población.

Entre los gustos que muestran la población en edad de trabajar están, en orden de preferencia, las siguientes alternativas: miscelánea (39\%), negocios caseros, como venta de gaseosas, rancho y víveres en microtiendas (28\%), manualidades y modistería $(15 \%)$, peluquerías $(10 \%)$, panadería $(8 \%)$. Las organizaciones de apoyo deben priorizar su orden y vocación para determinar las áreas estratégicas para su especialización y generación de capacidades productivas, representadas en gestión del conocimiento, el emprendimiento y generación de mayores ingresos.

\section{Caracterización de las capacidades de Ciencia Tecnología e Innovación CTI para la} distribución y comercialización.

Según la gráfica 1, de un total de 100 jefes de hogar, pertenecientes al barrio Ciudad Bonita, solo 6 tienen tablets o Ipad, 10 poseen conexión de Internet en su trabajo y 4 en su celular; así mismo, ninguno conoce los aplicativos digitales de Ciencia Tecnología e Innovación, para la distribución y comercialización: Agronet, Agronegocios, Celuagronet, Mipyme digital, ni otros cursos virtuales para aumentar sus ventas. Mientras que 34 tienen computador, 92 tienen celulares, 48 tienen conexión a Internet en su hogar.

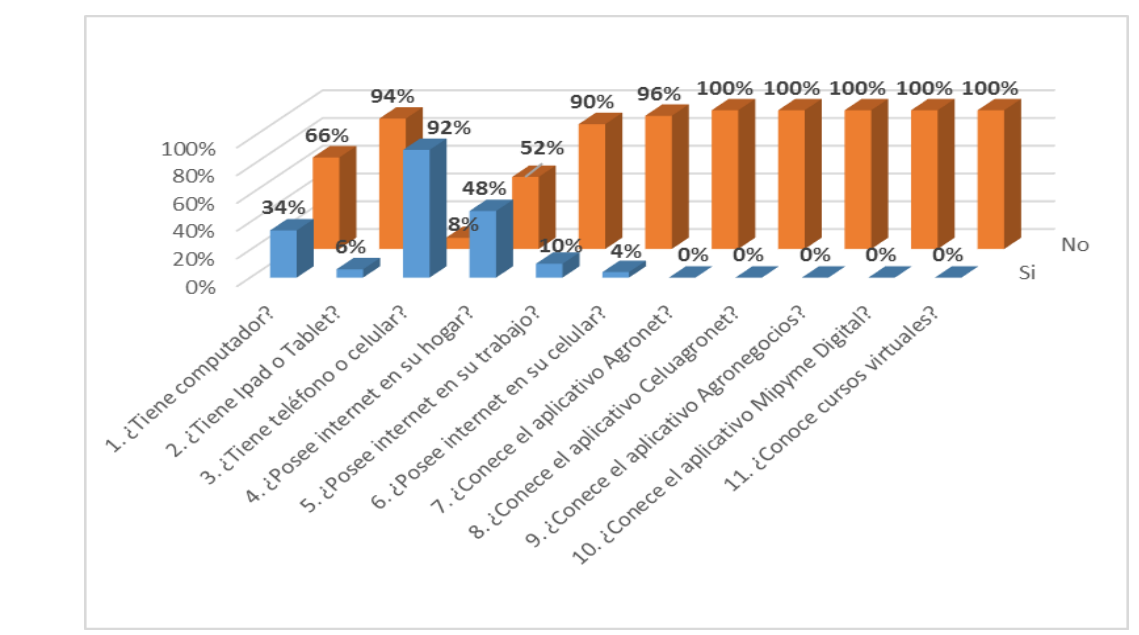

Grafico 1. Equipamiento y capacidades de Ciencia Tecnología e Innovación para la distribución y comercialización por jefe de hogar del barrio Ciudad Bonita de Soledad, Atlántico.

Nota. Fuente: elaboración propia con base al formato de caracterización de alfabetización digital de la Agencia de Desarrollo Rural (2018).

\section{Discusiones}

Los resultados obtenidos en esta investigación sobre la medición de las capacidades en los aspectos: a) productivos y sociales, b) económicos y financieros y c) comerciales y tecnológicos, coinciden con la metodología de la Universidad de Oxford, relacionada con la teoría de las capacidades económicas del premio nobel Sen (1985), la 
Elkyn Lugo-Arias, Luis Fernando Landázury-Villalba, Luis Escorcia-Márquez, Johanna Padilla-Ortiz

cual genera buenos indicadores multidimensionales, que muestran una mejor aproximación de la realidad del problema de la pobreza y la desigualdad en la acumulación de capital humano y generación de ingresos, frente a la cual deben intervenir eficiente y estratégicamente las instituciones gubernamentales y privadas, con políticas públicas efectivas, que sean inclusivas, equitativas y que generen oportunidades en las comunidades, las cuales requieren potencializar sus capacidades y generar ingresos de manera sostenible para su familia, de modo que disminuya la pobreza y aprendan a manejar sus finanzas personales y defenderse en la vida.

Esta teoría se está intentando aplicar en Colombia por parte del gobierno nacional, departamental y distrital, dado el éxito que ha tenido en Perú, con el posconflicto. De la misma manera, se están prestando servicios comunitarios directamente para impulsar a las familias por medio de los programas de salud, educación, capacitación gratuitos y responsabilidad social del Departamento para la Prosperidad Social [DPS], el Servicio Nacional de Aprendizaje SENA, universidades públicas y privadas, becas en educación de jóvenes en acción, Colciencias, apoyo económico y financiero para proyectos en actividades productivas, empleo, emprendimiento, comercialización y negocios mediante las Tecnologías de la información y la comunicación, que son incluyentes para la participación de las familias vulnerables mayores de 17 años.

Sin embargo, la teoría de Sen (1995) no se puede cumplir en su totalidad, dadas las condiciones que presenta el país, en donde el gobierno está mejorando las políticas públicas de sus instituciones, pero que no tiene la cobertura suficiente para atender a la mayoría de la población, dados los problemas de corrupción, los insuficientes recursos que destina el gobierno para el gasto público, el cual se está pensando invertir en el futuro para indemnizar a los desplazados por el posconflicto, los cuales están acostumbrados a recibir subsidios y se han vuelto improductivos, dado que no se esfuerzan para capacitarse y generar sus propios ingresos, por lo cual el gobierno está empezando a concientizarlos para que "aprendan a pescar" a través de los programas sociales y poder conducirlos hacia la ruta de la prosperidad.

El problema anterior se deriva de las teorías tradicionales, como: la de las Necesidades Básicas Insatisfechas (NBI), las Condiciones de Vida (CV), la Línea de Pobreza (LP) y mapas de pobreza, realizadas por Max, Elizalde y Hopenhayn (1986), Rawls (1995), Feres y Mancero (2001), Ponthieux y Concialdi (2001), Henninger y Snel (2002), Núñez y Ramírez (2002), Quintero y Mejía (2006) y Serafini (2015), las cuales no son multidimensionales, son menos optimistas y buscan disminuir el problema de la pobreza temporalmente, buscando resultados en la generación de ingresos, lo más rápido posible, sin importar que se potencialicen las actividades y se generen ingresos por medio de subsidios, que estancan los procesos.

Estas teorías tradicionales obedecieron a la preocupación que se vivió en su momento, con el afán de la Organización de la Naciones Unidas ONU de llegar a las familias en pobreza extrema presentadas en los países menos desarrollados. Actualmente, todos los autores coinciden en la medición de indicadores integrales o multidimensionales en todas las áreas, donde se garantice mejorar los resultados de bienestar general de esta población.

En tal aspecto, se buscó como propósito generar una verdadera conciencia entre la sociedad civil y las autoridades, de que la verdadera intención con este trabajo académico-investigativo no tuvo la finalidad de cuestionar la desidia estatal, la falta de gobernabilidad, y todos los problemas adyacentes que complementan esta problemática, sino aportar datos y evidencias que propicien la creación de un modelo de intervención social, que contempla crear 
Elkyn Lugo-Arias, Luis Fernando Landázury-Villalba, Luis Escorcia-Márquez, Johanna Padilla-Ortiz

alternativas productivas a estas familias, encaminadas a la dignificación de la calidad de vida, salud, economía y educación integral, de cada una de estas personas, que crecen en el seno familiar de hogares altamente afectados con esta deprimente realidad.

Por cuanto a lo anterior, quedó absolutamente claro que las capacidades socioeconómicas y las funciones humanas de las instituciones son herramientas que contribuyen benéficamente a reducir el problema de medición de los indicadores de pobreza multidimensional, de donde se deriva gran parte de la generación de valores económicos, originalmente representados en términos financieros y económicos, los cuales son la rentabilidad económica, progreso e innovación social y acumulación de conocimiento en la generación de sus propios recursos.

\section{Conclusiones}

Gracias al desarrollo de esta investigación, se ahondó en un estudio más profundo, que permitió conocer, evaluar y analizar, con rigurosidad, las debilidades y fortalezas que presentan estos barrios en materia de procesos productivos y su impacto socioeconómico teniendo en cuenta los factores de observación, que señala la teoría de las capacidades y funciones humanas de Sen, para hacer frente en gran parte a la situación de pobreza de las familias y a su mejoramiento en la calidad de vida. Por tanto, siguiendo con los objetivos y lineamientos trazados como propósito en este trabajo y articulando el análisis de la información documental recopilada, en conjunto con los resultados que se generaron a partir del trabajo de campo, se obtienen las conclusiones que se tratan a continuación.

Se registra una baja generación de ingresos en la población mayor de 17 años y los jefes de hogar encuestados en el barrio Ciudad Bonita ya que son muy pocos los que utilizan las estrategias de ahorro con otras entidades y grupos de ahorro, la mayoría de ellos manejan sus ingresos personalmente, sin intervención de una entidad bancaria. También se registra que han aumentado las deudas y los gastos de esta población, la cual aparece reportada por entidades financieras en data crédito y la Sifín, por lo cual requieren intervención de las instituciones del gobierno, fundaciones y cooperativas sociales, tanto nacionales como internacionales, en inclusión de programas de formación para el manejo de las finanzas y la generación de ingresos con proyectos productivos.

Existe la voluntad por parte de los jefes de hogar de participar en los programas de generación de ingresos por instituciones del Estado, por lo cual mostraron en su mayoría estar interesados en capacitarse en ventas y administración de negocios, así mismo están interesados en emprender su propio negocio, relacionado con venta de misceláneas, venta de víveres en microtiendas, manualidades, modistería, salones de belleza o peluquerías y panaderías. Por ende, el gobierno y las instituciones que velan por el bienestar de las localidades deben intervenir, fortalecer y potencializar las capacidades que tienen en este barrio vulnerable y abandonado para mejorar la situación económica del municipio de Soledad.

Por otro lado, la población en edad de trabajar presenta disponibilidad para realizar cualquier actividad y participar en los programas sociales y proyectos productivos, dado que en su mayoría están afiliados a un buen sistema de salud subsidiada, donde no han tenido ningún tipo de discapacidad, ni ninguna limitación grave. Sin embargo, existe en su mayoría un bajo nivel de educación en la mayoría de los jefes de hogar, con estudios de primaria e incluso analfabetas, por lo cual muchos están desempleados y trabajan como independientes, lo cual ha incidido en los problemas de desarrollo de la familia, tales como separación de parejas y soltería, por su difícil situación económica. 
Elkyn Lugo-Arias, Luis Fernando Landázury-Villalba, Luis Escorcia-Márquez, Johanna Padilla-Ortiz

Las personas que tienen estabilidad económica, en cambio, entre ellos personas capacitadas, asalariados y pensionados, se encuentran en una situación socioeconómica y productiva mejor, que le brinda cierto bienestar en su núcleo familiar.

\section{Referencias}

Agencia de Desarrollo Rural. (2018). Modelo de atención y prestación de servicios de apoyo a la comercialización. Recuperado de https://www.adr.gov.co/servicios/comercializacion/Paginas/alfabetizacion-digital-enfasiscomercial.aspx

Arrow, K., Bowles, S. \& Durlauf, S. (2018). Meritocracy and economic inequality. Princeton, New Jersey: University Press.

Botello, S. \& Quintero, L. (2012). Condiciones subjetivas de bienestar, felicidad y autopercepción de la pobreza en Colombia. CIFE, 14(21), 215-245. DOI: https://doi.org/10.15332/s2248-4914.2012.0021.02

Bravo, A. Schwartz, P. \& Drèze, J. (2014). Una gloria incierta. India y sus contradicciones. Madrid: Taurus.

Browning, M. \& Lusardi, A. (1996). Household Saving: Micro Theories and Micro Facts. Journal of Economic Literature, 34(4), 1797-1855. Recuperado de www.jstor.org/stable/2729595

Feres, J. \& Mancero, X. (2001). El método de las necesidades básicas insatisfechas y sus aplicaciones en América Latina. Santiago de Chile: Cepal.

Henninger, N. \& Snel, M. (2002). Where are the poor? Experiences with the development and use of poverty maps. Washington, DC, USA: World Resources Institute.

Henríquez-Fuentes, G., Lombana-Coy, J., González-Ariza, A., Higuera-Ojito, V., Landázury-Villalba, L., Rada-Llanos, J. \& Simancas-Trujillo, R. (2018). La gobernanza y su relación con la competitividad en una firma integrada a una cadena de suministro. Recuperado de http://bonga.unisimon.edu.co/handle/123456789/2086.

Landázury, L., Jaafar, H., Cristofani, M. \& Canales, R. (2018). Innovación y modelos de gerencia: su reflexión transformadora desde lo humano y el conocimiento. Revista espacios, 39(13). Recuperado de http://bonga.unisimon.edu.co/handle/123456789/1972

Landázury, L., Lechuga, J., Ferrer, F. \& Prieto, R. (2017). Innovación como eje transversal en los negocios internacionales. En Ziritt, G., Taboada, R., Prieto, F., Jiménez, L. \& Barboza, J. (Comp.). La producción de conocimiento desde el campo de las ciencias económicas, administrativas y contables (pp-275-304). Zulia: Fondo Editorial UNERMB.

Lugo, E., Landázury, L., Ferrer, F., Roncallo, A., Lugo, J., Jaafar, H. \& Ovallos, D. (2018). Productividad, competitividad y creación de valor del sector agroexportador: un enfoque de los costos, la responsabilidad social y la gestión ambiental en la Región Caribe. Barranquilla: Ediciones Universidad Simón Bolívar. 
Lugo, E., Sáenz, J. \& Lugo, J. (2018). La productividad como factor de competitividad de las exportaciones de aceite de palma del departamento del Magdalena Colombia, 2007-2015. Revista ciencia, saber y libertad, 13(1), 145-163. DOI: https://doi.org/10.18041/2382-3240/saber.2018v13n1.2088

Lugo, J. \& Lugo, E. (2018). Beneficios socio ambientales por potabilización del agua en los pueblos palafíticos de la Ciénaga Grande de Santa Marta-Colombia. Revista U.D.C.A, actualidad y divulgación científica, 21(1), 259264. DOI: https://doi.org/10.31910/rudca.v21.n1.2018.685

Lustig, N. \& Széley, M. (1998). México: Evolución económica, pobreza y desigualdad. En Ganuza, E. Taylor, L. \& Morley, S. Política macroeconómica y pobreza en América Latina y el Caribe. Madrid, España: Ediciones Mundi-Prensa.

Max-Neef, M., Elizalde, A., \& Hopenhayn, M. (1986). Desarrollo a escala humana: una opción para el futuro. Santiago de Chile: Biblioteca CF + S.

Michalos, A. (2017). What did Stiglitz, Sen and Fitoussi get right and what did they get wrong? Social Indicators Research, 102(1), 117-129.Recuperado de https://www.jstor.org/stable/41476470

Núñez, J. \& Ramírez, J. (2002). Determinantes de la pobreza en Colombia: Años recientes. Bogotá, Colombia: Naciones Unidas CEPAL.

Nussbaum, M. (2011). Creating Capabilities. The Human Development Approach. United States of America: Harvard University Press.

Pettit, P. (2001). Symposium on Amartya Sen's philosophy: 1 Capability and freedom: a defence of Sen. Economics \& Philosophy, 171), 1-20. DOI: https://doi.org/10.1017/S0266267101000116

Ponthieux, S. \& Concialdi, P. (2001). Bajos salarios y trabajadores pobres: una comparación entre Francia y Estados Unidos. Cuadernos de Relaciones Laborales, 18(1), 173-203. Recuperado de http://revistas.ucm.es/index.php/CRLA/article/viewFile/CRLA0101120173A/32452

Quintero, L. \& Mejía, A. (2006). Percepción subjetiva de los pobres: Una alternativa a la medición de la pobreza. Reflexión Política, 8(15), 26-40. Recuperado de https://dialnet.unirioja.es/servlet/articulo?codigo=2134837

Rawls, J. (1995). Teoría de la Justicia. México: Fondo de Cultura Económica.

Robeyns, I. (2005). The Capability Approach: a theoretical survey. Journal of Human Development, 6(1), 93-114. DOI: https://doi.org/10.1080/146498805200034266

Sen, A. (1985). Commodities and Capabilities. Amsterdam: North-Holland.

Sen, A. (1987). The Standard of Living. Cambridge: Cambridge University Press. 
Sen, A. (1990). Development as capability expansion. In Hollis, C. \& Thirukodikaval, S. (eds.) Handbook of Development Economics (pp. 10-26) North Holland: Elsevier Science Publishers.

Sen, A. (1995). Nuevo Examen de la Desigualdad. Madrid: Alianza Editorial.

Sen, A. (2018). Collective choice and social welfare. United States: Harvard University Press.

Serafini, V. (2015). Pobreza, oportunidades económicas desiguales y género [Documento de trabajo]. Asunción: ONU Mujeres, PNUD.

Stiglitz, J., Sen, A. \& Fitoussi, J. P. (2010). Report by the commission on the measurement of economic performance and social progress. Paris: Commission on the Measurement of Economic Performance and Social Progress. Recuperado de https://ec.europa.eu/eurostat/documents/118025/118123/Fitoussi+Commission+report

Sugden, R. (1993). Welfare, resources, and capabilities: a review of inequality reexamined by Amartya Sen. Journal of Economic Literature, 31(4), 1947-1962. Recuperado de https://www.jstor.org/stable/2728332

Universidad Minuto de Dios. (2016). Estructura organizacional de la Proyección Social. Diseño de un modelo de intervención Psicosocial y económico para la población vulnerable del municipio de Baranoa: Barrio La Ceiba y el municipio de Soledad: Barrio Ciudad Bonita. Recuperado de https://n9.cl/7xz9

Wells, T. (2012). Sen's Capability Approach. In Feiser, J. \& Dowden, B. (eds.). Internet Encyclopedia of Philosophy. Recuperado de https://www.iep.utm.edu/sen-cap/ 